Check for updates

Cite this: RSC Adv., 2018, 8, 22806

Received 26th April 2018

Accepted 13th June 2018

DOI: $10.1039 / c 8 r a 03598 a$

rsc.li/rsc-advances

\section{Stimuli-responsive fluorescence switching of cyanostilbene derivatives: ultrasensitive water, acidochromism and mechanochromism $\uparrow$}

\begin{abstract}
Bin Wang ${ }^{\text {ab }}$ and ChunYing Wei (iD)*a
A novel donor- $\pi$-acceptor structure stimuli-responsive fluorescent material of ( $Z$ )-2-(4' - (diphenylamino)[1,1'-biphenyl]-4-yl)-3-(pyridin-2-yl)acrylonitrile (oN-TPA) was designed and synthesized, with the cyanogroup and pyridine as the acceptors (A) and triphenylamine as the donor (D). oN-TPA exhibits an obvious solvatochromic effect and the excited state is confirmed to be a hybridized local and charge-transfer (HLCT) state that simultaneously possesses the locally-excited (LE) state and charge transfer (CT) state characters. The LE state ensures relatively high fluorescence efficiency while the CT state provides multistimuli responsive fluorescence behaviors because it is easily tuned by the surrounding environment. Firstly, oN-TPA exhibits "on-off-on" fluorescence properties in the mixture of water/tetrahydrofuran (THF) with the increasing water content. For the "on-off" part, a good linear relationship between fluorescence intensity and water fraction is achieved, which is ascribed to the synergistic effect of protons in water and intramolecular charge-transfer (ICT) effect depending on solvent polarity. The "offon" part demonstrates the aggregation-induced enhanced emission (AIEE) character of oN-TPA. Secondly, oN-TPA can be used as a protonic acid sensor to detect trifluoroacetic acid (TFA) in solvent and $\mathrm{HCl}$ vapour in the solid state due to the binding of the proton to the pyridine group. Finally, oN-TPA presents remarkable and reversible mechanochromic fluorescence switching between $552 \mathrm{~nm}$ and $642 \mathrm{~nm}$ (90 nm red-shift) during the pressurizing-depressurizing process. This work not only comprehensively demonstrates the stimuli-responsive fluorescence behaviors of oN-TPA, but also provides a $D-\pi-A$ structure fluorescent material possessing potential applications in detection and sensing with remarkable fluorescence changes.
\end{abstract}

\section{Introduction}

Stimuli-responsive fluorescent materials have recently attracted more and more attention due to their potential applications as sensors, switches, and recording media in the past few years. ${ }^{1}$ Recently, various organic materials with efficient and reversible fluorescence behaviors have been developed, responding to light, ${ }^{2}$ mechanical force,${ }^{3}$ temperature $,{ }^{4} \mathrm{pH},{ }^{5}$ water, ${ }^{6}$ and solvent vapors. ${ }^{7}$ In the design of stimuli-responsive materials, a high solid-state efficiency is necessary for achieving obvious changes in fluorescence behavior towards various external stimuli. However, most luminescent materials suffer from the effects of aggregation-caused quenching (ACQ), which limits the application of chromic materials. A phenomenon named

${ }^{a}$ Key Laboratory of Chemical Biology and Molecular Engineering of Ministry of Education, Institute of Molecular Science, Shanxi University, Taiyuan 030006, PR China.E-mail:weichuny@sxu.edu.cn

${ }^{b}$ School of Basic Medical Sciences, Shanxi Medical University, Taiyuan 030001, P. R. China. Tel: (+)86-351-7010699

$\uparrow$ Electronic supplementary information (ESI) available. See DOI: 10.1039/c8ra03598a aggregation-induced enhanced emission (AIEE) appeared in 2001, which demonstrated enhanced emission in the aggregate state for the restriction of intramolecular rotations (RIR) ${ }^{8}$ From then on, more and more AIEE character luminescent materials have been developed and studied in organic light-emitting diodes (OLEDs), ${ }^{9}$ luminescent sensors, $^{\mathbf{1 0}}$ biomedical imaging, ${ }^{11}$ and smart materials. ${ }^{12}$ Except for the typical AIEE group of tetraphenylethene (TPE), many other systems have also been found to possess AIEE character. ${ }^{13}$

Charge transfer (CT) fluorescent complexes usually consist of electron donor (D) and electron acceptor (A) parts, which have been extensively exploited in OLEDs, ${ }^{\mathbf{1 4}}$ organic photovoltaics (OPVs), ${ }^{15}$ field-effect transistors (FETs), ${ }^{16}$ nonlinear optics, and fluorescent chemosensors. ${ }^{17}$ CT molecules always show the strong solvatochromic effect, and their photoluminescence (PL) emissions exhibit a large red shift with the increase of solvent polarity. Therefore, the fluorescence emissions of CT-materials are greatly dependent on their excited states, which can be easily tuned by the surrounding environment, such as external stimulus. However, the strong CT-state usually causes a serious decrease in PL efficiency due to the small orbital overlap. Ma et al. proposed a named hybridized 
local and charge-transfer (HLCT) ${ }^{18}$ state method to realize high fluorescence efficiency $\left(\eta_{\mathrm{PL}}\right)$, and this excited state simultaneously possesses the locally-excited (LE) state and CT state characters. The LE component ensures relatively high fluorescence efficiency, and the CT component provides multi-stimuli responsive fluorescent behavior, which may provide a new strategy to achieve high-efficient and high-contrast stimuli responsive materials.

Herein, we report a D- $\pi-\mathrm{A}$ structure molecule of $(Z)-2-\left(4^{\prime}-\right.$ (diphenylamino)-[1,1'-biphenyl]-4-yl)-3-(pyridin-2-yl)-acrylonitrile (oN-TPA) (Scheme 1), which exhibits the multi-stimuli responsive fluorescent properties. Solvatochromic experiments exhibit a large red-shift from $468 \mathrm{~nm}$ in low polar hexane to $615 \mathrm{~nm}$ in high polar acetonitrile. Combined with theoretical calculation and Lippert-Mataga model, ${ }^{19}$ the excited state is confirmed to be a HLCT state. The photophysical properties of oN-TPA in the mixture of THF/water demonstrate a pronounced AIEE effect. Moreover, it shows ultra-sensitivity PL response to water in THF, which can be used as a detector for water in organic solvents. The color and intensity of fluorescence produce the substantial changes by protonation, thus enabling it to work as a fluorescent $\mathrm{pH}$ sensor in both solution and solid states. At the same time, the switchable fluorescence of $\boldsymbol{o N}$-TPA in the solid state is achieved during pressurizing-depressurizing process. Therefore, this work provides a comprehensive insight into the stimuli-responsive fluorescence behavior of a HLCT state luminescent material and a new strategy to achieve high efficient and easily tuned stimuli-responsive materials.

\section{Experimental section}

\subsection{Constant-pressure experiments}

The ${ }^{1} \mathrm{H}$ NMR spectra were recorded by a Bruker Avance III 500 $\mathrm{MHz}$ spectrometer with DMSO- $d_{6}$ as solvent and tetramethylsilane (TMS) as standard. The Shimadzu UV-2600 spectrophotometer (Japan) and SENS-9000 (GildenPhotonics, England) spectro-photometer were used to record UV-vis and PL spectra, respectively, and a solution of the sample $\left(\mathrm{ca} \cdot 10^{-5} \mathrm{M}\right)$ in a $1 \mathrm{~cm}$ quartz cuvette was used in the measurement. Digital photographs were taken by 550D (Canon, Japan) digital cameras. We used spectrometer C11347 (Hamamatsu, Japan) to measure their absolute fluorescence quantum yields. Powder XRD measurements were conducted in the range $5<2 \theta<40$ (PANalytical, Netherlands). X-ray crystallographic intensity data were collected using a Bruker APEX-II CCD, Gemini Ultra CCD diffractometer equipped with a graphite monochromated Enhance (Mo) X-ray source $(\lambda=0.71073 \AA)$.

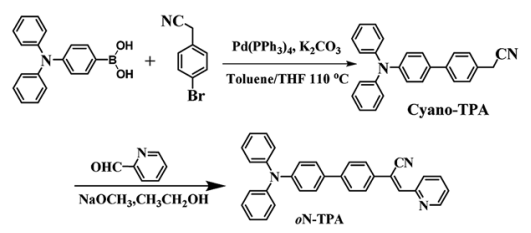

Scheme 1 Synthetic route to oN-TPA.

\subsection{High-pressure experiments}

The crystal was placed in the hole of a T301 steel gasket, A $4: 1$ mixture of methanol and ethanol was used as a pressure transmitting medium (PTM). We conducted in situ steady-state PL measurements on an Ocean Optics QE65000 spectrometer in the reflection mode. Excitation source is the $355 \mathrm{~nm}$ line of a DPSS laser with a spot size of $20 \mathrm{~mm}$. The diamond anvil cell (DAC) containing the sample was put on a Nikon fluorescence microscope to focus the laser on the sample. Optical photographs of the compressed $\boldsymbol{o N}$-TPA samples were taken by an imaging camera (Canon EOS 5D Mark II) equipped on the fluorescence microscope. In situ transient-state PL was tested with a laser strobe spectro-fluorometer (produced by photon technology instruments) coupled to an inverted fluorescence microscope (Nikon Ti-U).

\subsection{Lippert-Mataga model}

The influence of solvent environment on the optical property of oN-TPA can be understood using the Lippert-Mataga equation: ${ }^{19}$

$$
h c\left(\nu_{\mathrm{a}}-\nu_{\mathrm{f}}\right)=h c\left(\nu_{\mathrm{a}}^{0}-\nu_{\mathrm{f}}^{0}\right)-\frac{2\left(\mu_{\mathrm{e}}-\mu_{\mathrm{g}}\right)^{2}}{a^{3}} f(\varepsilon, n)
$$

where $f$ is the orientational polarizability of solvents, $\nu_{\mathrm{a}}^{0}-$ $\nu_{\mathrm{f}}^{0}$ corresponds to the Stokes-shift when $f$ is zero. $\mu_{\mathrm{e}}$ and $\mu_{\mathrm{g}}$ are the dipole moments of the excited state and ground state, respectively, $a$ is the solvent cavity (Onsager) radius, $\varepsilon$ is the solvent dielectric, and $n$ is the solvent refractive index.

\subsection{Quantum chemical calculations}

All the density functional theory (DFT) calculations were carried out using Gaussian 09 (version D.01) package on a Power Leader cluster. The excited state geometry was optimized by timedependent density functional theory (TD-DFT) with the B3LYP functional at the basis set level of $6-31 \mathrm{G}(\mathrm{d}, \mathrm{p})$. The emission properties were obtained using TD-M06-2X/6-31G(d,p) at the excited state geometries.

\subsection{Synthesis and structural characterization of $o N-T P A$}

Solution of cyano-TPA ( $1.8 \mathrm{~g}, 5 \mathrm{mmol})$ prepared according to the previous literatures ${ }^{18 d}$ and 4-formylbenzonitrile ( $\left.0.65 \mathrm{~g}, 6 \mathrm{mmol}\right)$ in anhydrous EtOH (chromatographically pure, $30 \mathrm{ml}$ ) was treated with $\mathrm{NaOCH}_{3}(0.12 \mathrm{~g}, 2 \mathrm{mmol})$, stirred at room temperature for over $2 \mathrm{~h}$, cooled to $0{ }^{\circ} \mathrm{C}$, and filtered. The precipitate was repeatedly washed with $\mathrm{EtOH}$ to give the desired oN-TPA powders $(2.2 \mathrm{~g}, 90 \%)$. oN-TPA: ${ }^{1} \mathrm{H}$ NMR $(500 \mathrm{MHz}$, DMSO- $d_{6}$ see Fig. S1, ESI $\left.\dagger\right) \delta 8.76(\mathrm{~d}, J=4.0 \mathrm{~Hz}, 1 \mathrm{H}), 8.10(\mathrm{~s}, 1 \mathrm{H})$, $7.95-7.99(\mathrm{~m}, 1 \mathrm{H}), 7.90(\mathrm{~d}, J=8.5 \mathrm{~Hz}, 2 \mathrm{H}), 7.82(\mathrm{~d}, J=8.5 \mathrm{~Hz}$, $2 \mathrm{H}), \delta 7.78(\mathrm{~d}, J=9.0 \mathrm{~Hz}, 1 \mathrm{H}), 7.69(\mathrm{~d}, J=9.0 \mathrm{~Hz}, 2 \mathrm{H}), 7.47-7.49$ $(\mathrm{m}, 1 \mathrm{H}), 7.35(\mathrm{t}, J=8.0 \mathrm{~Hz}, 4 \mathrm{H}), 7.05-7.11(\mathrm{~m}, 8 \mathrm{H}) ;{ }^{13} \mathrm{C} \mathrm{NMR}(500$ MHz, DMSO- $\left.d_{6}\right) \delta=154.4,150.1,147.5,140.3$, 136.8, 133.4, $129.4,127.7,127.1,126.8,124.7,124.2,123.5,123.3,117.4$ and 114.6, $\mathrm{MS}\left(\mathrm{EI}^{+}\right): m / z 449.2$. 


\section{Results and discussion}

\subsection{Solvatochromic effect}

The oN-TPA possesses a typical $\mathrm{D}-\pi-\mathrm{A}$ structure, in which the triphenylamine group acts as the electron donor part against the acceptor of the cyano-group and pyridine. Since the photophysical properties of $\mathrm{D}-\pi-\mathrm{A}$ molecules are dependent on the solvent polarity, the absorption and PL spectra of $\boldsymbol{o N}$-TPA were measured in the different solvents (Fig. S2, ESI $\dagger$ and Fig. 1), and the detailed data shown in Table S1, ESI. $\dagger$ The absorption spectra of $\boldsymbol{o N}$-TPA display the minor changes in both shape and position in the different solvents (Fig. S2 $\dagger$ ). In contrast, with the increase of solvent polarity, the emission peak demonstrates a gradual red-shift from $469 \mathrm{~nm}$ in hexane to $650 \mathrm{~nm}$ in acetonitrile, exhibiting a strong solvatochromic effect (Fig. 1a-c). At the same time, the vibronic fine structure disappears and emission band broadens from low polarity to high polarity solvents, which illustrates an obvious intra-molecular charge-transfer (ICT) character in excited state. In addition, a clear decreasing trend of the $\eta_{\mathrm{PL}}$ in the different solvents is observed, which is ascribed to the CT effect depending on solvent polarity. ${ }^{19,20}$

To evaluate the solvatochromic effect, the Lippert-Mataga model is applied to estimate the dipole moment $\left(\mu_{\mathrm{e}}\right)$ of the excited state. The plot of Stokes shift against orientation polarizability $(\Delta f)$ is shown in Fig. 1d. Notably, two linear relationships can be observed, indicating two different excited state properties in low and high polar solvents, respectively. The dipole moment, $\mu_{\mathrm{e}}$, is calculated to be $19.8 \mathrm{D}$ in high polarity solvents, which is near the typical CT compound DMABN with $\mu_{\mathrm{e}}$ of $23 \mathrm{D}^{21}$ The

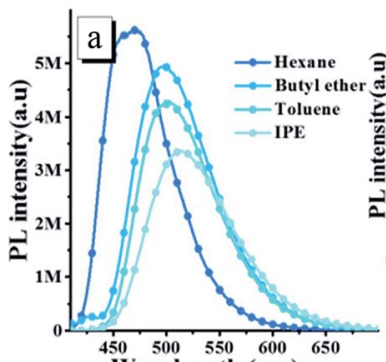

Wavelength $(\mathbf{n m})$
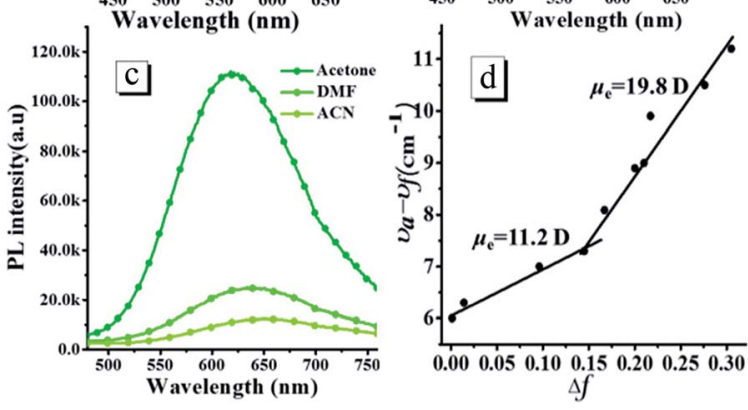

Fig. 1 The fluorescence spectra $(a-c)$ of oN-TPA measured in the different solvents with the increasing polarity (the orientational polarizability of solvent, $\Delta f, n$-hexane: 0.0012 ; butyl ether: 0.096 ; toluene: 0.014; isopropyl ether (IPE): 0.145; tetrahydrofuran (THF):0.210; ethyl acetate (EAC): 0.200; dichloromethane (DCM): 0.217; N,N-dimethylformamide (DMF): 0.276; acetone: 0.284; and acetonitrile (ACN): 0.305), excitation at $400 \mathrm{~nm}$, slit width 3; (d)the fitted linear correlation of the Stokes shift $\left(\nu_{a}-\nu_{f}\right)$ as a function of solvent polarity for oN-TPA in the low- and high-polarity regions. rapid decrease of PL quantum yield from 59.6\% in THF to $0.8 \%$ in acetonitrile also illustrates a CT-dominant excited state in high-polarity solvents. While in low-polarity solvents, the dipole moment is calculated to be $11.2 \mathrm{D}$, which is obviously smaller than that of CT state in high polarity solvents. However, the red shift of $39 \mathrm{~nm}$ from 0.0012 in hexane to 0.145 in isopropyl ether indicates that the CT character still exists for $\mathrm{S}_{1}$ state. ${ }^{18}$ Meanwhile, a vibration fine structure and relatively high $\eta_{\mathrm{PL}}$ in hexane demonstrate the existence of LE component. Thus, the $S_{1}$ state in low-polarity solvents contains CT and LE components simultaneously. The $\mu_{\mathrm{e}}$ of $11.2 \mathrm{D}$, which is smaller than DMABN but larger than the LE compound about $8 \mathrm{D},{ }^{21}$ is ascribed to the HLCT state character. The LE component in HLCT state ensures a relatively high PL efficiency, and the CT component in the excited state results in a large Stokes shift from low polar solvents to high polar solvents, which is sensitive to the external stimuli.

\subsection{Aggregation-induced enhanced emission}

To better understand the nature of excited states, we calculated and analyzed the $\mathrm{S}_{1} \rightarrow \mathrm{S}_{0}$ natural transition orbital (NTO) ${ }^{22}$ of oN-TPA as depicted in Fig. 2. For the $\mathrm{S}_{1}$ state, the hole is delocalized over the whole molecule, while the particle is mainly localized at the diphenylethylene group with a small part of benzene on the TPA group. The separated orbitals provide a CT component with large dipole moment. In addition, orbital overlaps on acceptor provide a LE component, which ensures a relatively high radiative-transition rate with the oscillator strength $(f)$ of 1.5188. NTO analysis indicates the coexistence of LE and CT components. The twist angle between donor and acceptor is $19.5^{\circ}$. Benefiting from the proper twist angle, a balance of LE and CT component is achieved.

To investigate the AIEE properties of $\boldsymbol{o N}$-TPA, we measured the absorption and PL spectra in the water/THF mixture with different water fractions (Fig. S3, ESI $\dagger$ and Fig. 3). The fluorescence emissions demonstrate a slight red-shift and the intensities sharply decrease with gradual addition of water into the THF solution up to $70 \%(\mathrm{v} / \mathrm{v})$, which can be ascribed to the enhanced ICT effect due to the increased solvent polarity. However, when the water fraction increases up to $80 \%$, the aggregates appears, which can be verified by the lifted spectral tails from their absorption spectra. In addition, an obvious enhancement of the fluorescence intensity is observed because of the restriction in intramolecular rotations (RIR). As the water fractions reaches $90 \%$, the fluorescence emission is further enhanced. Another reason for this AIEE behavior could be that the ICT process is effectually prevented, which is also helpful for the emission of light. Therefore, the emission maxima exhibit a blue-shift from $576 \mathrm{~nm}$ to $560 \mathrm{~nm}$.

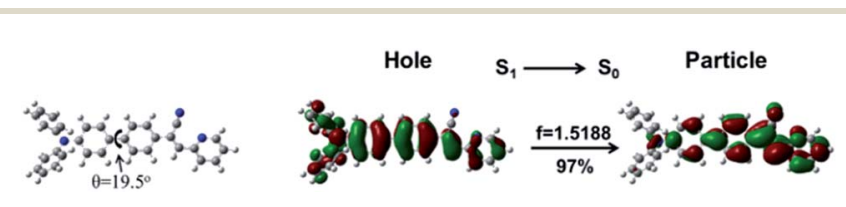

Fig. 2 The $S_{1} \rightarrow S_{0}$ NTO of oN-TPA. Herein, $f$ represents the oscillator strength, and the weights of hole-particle are given for the $S_{1} \rightarrow S_{0}$ excitation. 


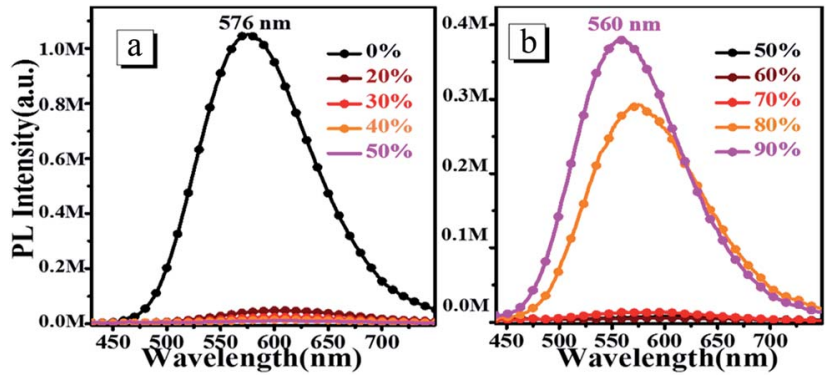

Fig. 3 The steady-state fluorescence spectra of $10 \mu \mathrm{M}$ oN-TPA in THF alone $(0 \%)$ and THF-water mixture with water fractions from $20 \%$ to $90 \%(v / v)$.

\subsection{Water sensing}

Based on the above results, the fluorescence emission of $\boldsymbol{o N}$ TPA is highly sensitive to water, thus we further investigate its emission behaviour in THF by gradually increasing the content of water. Fig. 4a shows the fluorescence spectra of $\boldsymbol{o N}$-TPA in THF with increasing the water fraction from 0 to $10 \%(\mathrm{v} / \mathrm{v})$ in mixture solvents. In pure THF, oN-TPA exhibits a high PL efficiency of 59.6\%, benefiting the full hybridization of LE and CT component. With the increase of water content, a significant decrease in the fluorescence intensity is observed, along with the red shift from $573 \mathrm{~nm}$ to $601 \mathrm{~nm}$. It is ascribed to the synergistic effect of proton in water and ICT in mixture solvent. The fluorescence intensity is quenched by $61.9 \%$ when water content rises up to $3 \%$. Especially, there is nearly no fluorescence emission when the water content reaches $7 \%$. More importantly, a good linear relationship between fluorescent intensity $\left(I_{0}-I\right) / I$ and water fraction is achieved when water fraction below 1\% (Fig. S4, ESI $\dagger$ ). The Stern-Volmer quenching constant $\left(K_{\mathrm{SV}}\right)$ is $0.52 \mathrm{M}^{-1}$, and the detection limit for water is $0.017 \%$ (231 ppm) in THF (Fig. 4b). Such a low detection limit indicates the high sensitivity of $\boldsymbol{o N}$-TPA to water in THF. The results above suggest that $\boldsymbol{O N}$-TPA can be used as a fast and effective PL probe to quantitatively detect low-level water in organic solvents.

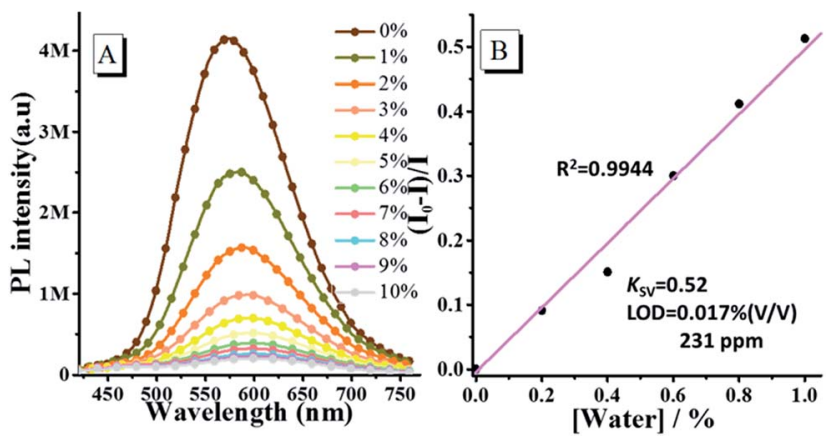

Fig. 4 (A) PL spectra of $10 \mu \mathrm{M}$ oN-TPA in THF/water mixture solvents with water fraction from 0 to $10 \%(\mathrm{v} / \mathrm{v})$ (excitation at $400 \mathrm{~nm}$, slit width 3). (B) The plot of $\left(I_{0}-I\right) / /$ with water fractions from 0 to $10 \% . I_{0}$ and $I$ represents the intensity in the absence and presence of water, respectively.

\subsection{Sensing properties toward protonic acids}

3.4.1 Response to $\mathbf{p H}$ in solvent. $\boldsymbol{o N}$-TPA may be used as a sensor for detecting $\mathrm{H}^{+}$, because the pyridine unit can bind with a proton to form a cation. To investigate its response to $\mathrm{pH}$, the PL spectra of $\boldsymbol{o N}$-TPA in THF-water mixtures with volume ratios of $1: 9$ at various $\mathrm{pH}$ were measured, and the $\mathrm{pH}$ was tuned by adding trifluoroacetic acid (TFA) concentration. As shown in Fig. 5a, after adding TFA for 3 minutes, the PL spectrum at $\mathrm{pH} 0.98$ is almost a flat line parallel to the abscissa because of its transformation to the weakly emissive $\boldsymbol{o N}$-TPAH ${ }^{+}$ under this acidic condition. ${ }^{23}$ When the $\mathrm{pH}$ increases to 1.86 , there is still a weak fluorescence after 3 minutes (Fig. 5b). With the time getting longer, the $\boldsymbol{o N}$-TPA molecule further undergoes protonation and its emission intensity gets weaker. As the $\mathrm{pH}$ further increases to 3.34 and 5.53 (Fig. 5c and d), the effect of proton quench reduces because of the progressive decrease in the population of $\boldsymbol{o N}-\mathbf{T P A H}^{+}$in solution. The extent of transformation of $\boldsymbol{o N}$-TPA to $\boldsymbol{o N}-\mathbf{T P A H}^{+}$at the different $\mathrm{pH}$ values can also be followed by UV-vis absorption analysis (Fig. S5, ESI $\dagger$ ). With the $\mathrm{pH}$ reduced from 4.52 to 0.96 , the absorption peak at $374 \mathrm{~nm}$ nearly disappears and the band at $450 \mathrm{~nm}$ gradually increases, which should be ascribed to the formation of strong CT-state due to the increased electron withdrawing ability of protonated pyridine. At $\mathrm{pH}$ 4.52, a weak shoulder peak nearly disappears, showing that no protonation occurs at such a $\mathrm{pH}$ value.

3.4.2 Response to $\mathbf{H C l}$ vapor in solid state. To determine the response towards $\mathrm{HCl}$ vapour in the solid state, the crystalline powder is exposed to $\mathrm{HCl}$ vapor for several minutes. Fig. 6 shows the time dependence of steady-state PL spectra of

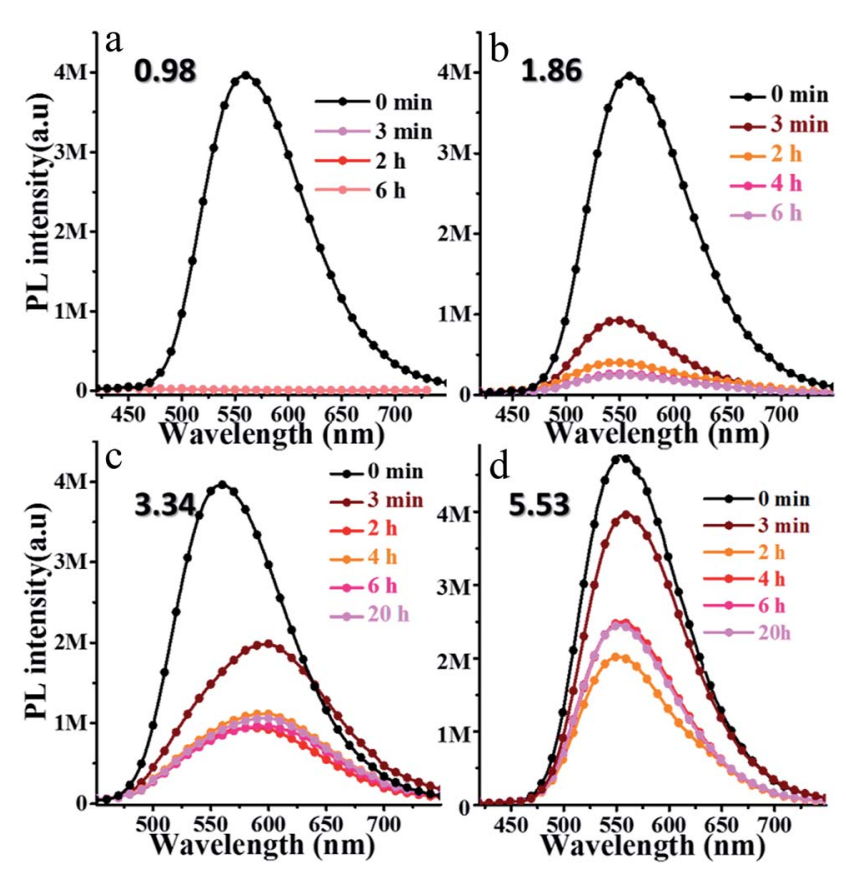

Fig. 5 Time dependence of fluorescence spectra of $10 \mu \mathrm{M}$ oN-TPA after adding the different TFA concentration to tune the $\mathrm{pH}$ values of solution to 0.98 (a), 1.86 (b), 3.34 (c), and 5.53 (d) in THF-water mixtures with volume ratios of $90 \%$ of water to THF (excitation at $400 \mathrm{~nm}$, slit width 3). 

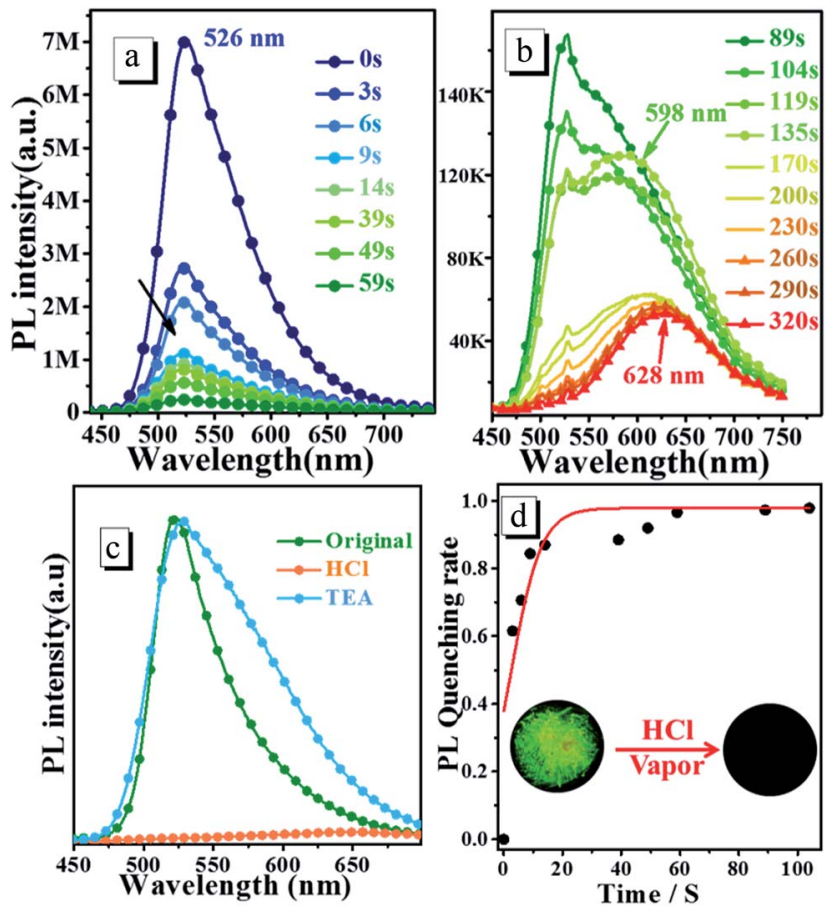

Fig. 6 (a) and (b) Time dependence of steady-state fluorescence spectra of oN-TPA crystalline powders upon consecutive exposure to $\mathrm{HCl}$ vapors, (c) PL spectra of oN-TPA crystalline powders fumed by $\mathrm{HCl}$ and then TEA vapor, (d) time-course of PL quenching rate of oN-TPA original powders exposed to $\mathrm{HCl}$ vapors.

oN-TPA crystalline powders upon exposure to $\mathrm{HCl}$ vapour (60 ppm). The original crystalline powder exhibits a green emission at $526 \mathrm{~nm}$ and a relatively high $\eta_{\mathrm{PL}}$ of $77.2 \%$, due to the AIEE effect. Upon exposed to $\mathrm{HCl}$ vapour, the fluorescence intensity significantly reduces due to the protonated pyridyl rings (see Fig. 6c). More than $85 \%$ of the fluorescence is quenched at $14 \mathrm{~s}$. With the increasing exposing time in $\mathrm{HCl}$ vapour, another new peak at $598 \mathrm{~nm}$ appears as depicted in Fig. 6a and b. The quenching reaches equilibration about $170 \mathrm{~s}$ with the quenching rate of approximately $98 \%$, that is, the population of $\boldsymbol{o N}$ $\mathbf{T P A H}^{+}$remains the same with the change of time. Finally, at $320 \mathrm{~s}$ there is a red emission band at $628 \mathrm{~nm}$ that should be ascribed to the strong CT emission from oN-TPAH ${ }^{+}$. Timecourse of PL quenching rate is shown in Fig. 6d, the quenching rate is over $95 \%$ after $60 \mathrm{~s}$. Furthermore, the reversibility experiment is carried out by exposing protonated crystalline powder to triethylamine (TEA) at room temperature. It is found that the emission maximum at $628 \mathrm{~nm}$ is quenched and the peak at $526 \mathrm{~nm}$ reappeared after a few minutes (see Fig. 6c). The recovered green fluorescence should be attributed to the deprotonation of pyridinium in $\boldsymbol{o N}-\mathbf{T P A}-\mathrm{HCl}$ by TEA to produce $\boldsymbol{o N}$-TPA and $\mathrm{NH}_{4} \mathrm{Cl}$. The crystalline powder after exposed to $\mathrm{HCl}$ vapor, significantly turns to red. In $365 \mathrm{~nm}$ UV light as shown in Fig. 6d, the green fluorescence disappears after protonation. This result demonstrates that oN-TPA could be used as a "naked-eye" and reversible sensing material for detecting $\mathrm{HCl}$ in practical applications.

The doped film is more favorable to application, because it possesses higher luminescence efficiency of $82.5 \%$ benefiting from the rigid environment and higher sensitivity to $\mathrm{HCl}$ vapor for its larger area to protonation. $\boldsymbol{o N}$-TPA is dispersed into PMMA matrix using $\mathrm{CHCl}_{3}(5 \%$, dye/PMMA, w/w) dried on neat quartz plate to obtain doped film. In the natural light, it presents yellow (see Fig. 7E), and it performances a bright yellow emission under $365 \mathrm{~nm}$ UV light (see Fig. 7F). Exposed to $\mathrm{HCl}$ vapor, the film rapidly changes from yellowish green to red under natural light (see Fig. 7A). The emission intensity under $365 \mathrm{~nm}$ UV light is quenched quickly with exposure time, fluorescence quenching more than $95 \%$ at $19 \mathrm{~s}$, and no fluorescent signal can be measured after 24 s (see Fig. 7B). Photographs of original and protonated film-doped in natural light and $365 \mathrm{~nm}$ UV light are given out for comparison (see Fig. 7C and D). These results indicate that $\mathrm{HCl}$ is an efficient medium for quenching the fluorescence of $\boldsymbol{o N}$-TPA, which can be used to quickly detect $\mathrm{HCl}$ vapor.

\subsection{Mechanofluorochromic experiments}

3.5.1 Mechanical grinding experiments. The $\boldsymbol{o N}$-TPA is also sensitive to mechanical grinding and shows reversible fluorescence changes. As depicted in Fig. 8, the original crystalline powders exhibit a green fluorescence at $524 \mathrm{~nm}$. Upon grinding, it displays a yellow emission with peak at $550 \mathrm{~nm}$ with the $\eta_{\mathrm{PL}}$ of $47.2 \%$. The amorphous state shows a broadened PL spectrum and a red shift of $26 \mathrm{~nm}$. It is further confirmed by identical their PXRD patterns (Fig. S6 $\dagger$ ). Compared to original sample, the ground powder displays significantly weakened and broadened peaks, indicating that the crystal structure is changed after grinding. Fuming with alcohol vapours, the fluorescence color recovers to green and the PXRD also recovers to original diffraction peaks, demonstrating the reversible switch between the crystalline powders and amorphous powders resulting in mechanochromic process. As depicted in Fig. S7, $\dagger$ the differential scanning calorimetry (DSC) thermogram of the ground powders revealed a clear endothermic peak at $106{ }^{\circ} \mathrm{C}$, which was attributed to glass transition temperature $\left(T_{\mathrm{g}}\right)$. However, the exothermic peak usually observed in other mechanochromic materials, did not appear. ${ }^{20}$ The result revealed that the phase transition (from amorphous to crystalline), was not achieved by heating treatments, which coincided with the experimental results.

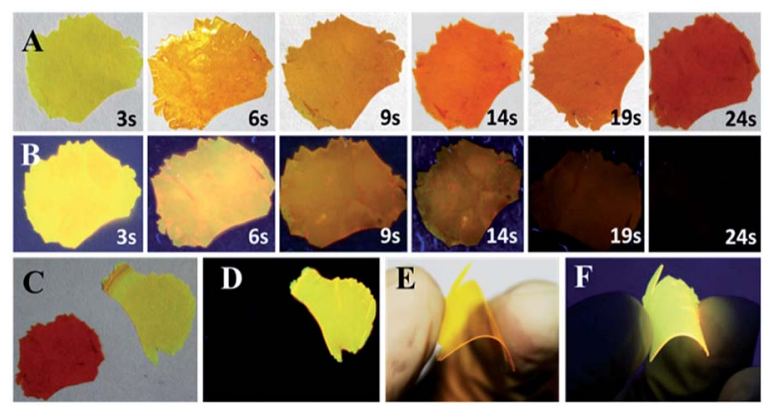

Fig. 7 Photographs of the dye (oN-TPA, 5\% wt/wt)-doped polymers (PMMA) film under $365 \mathrm{~nm}$ UV light (B, D and F) and natural light ( $A, C$ and $E$ ): ( $A$ and $B$ ) time dependence of the doped film upon consecutive exposure to $\mathrm{HCl}$ vapour (50 ppm), ( $C$ and $\mathrm{D}$ ) the doped films before/ after (final state, $10 \mathrm{~min}$ ) exposure to $\mathrm{HCl}$ vapor, (E and F) flexible thinfilm. 


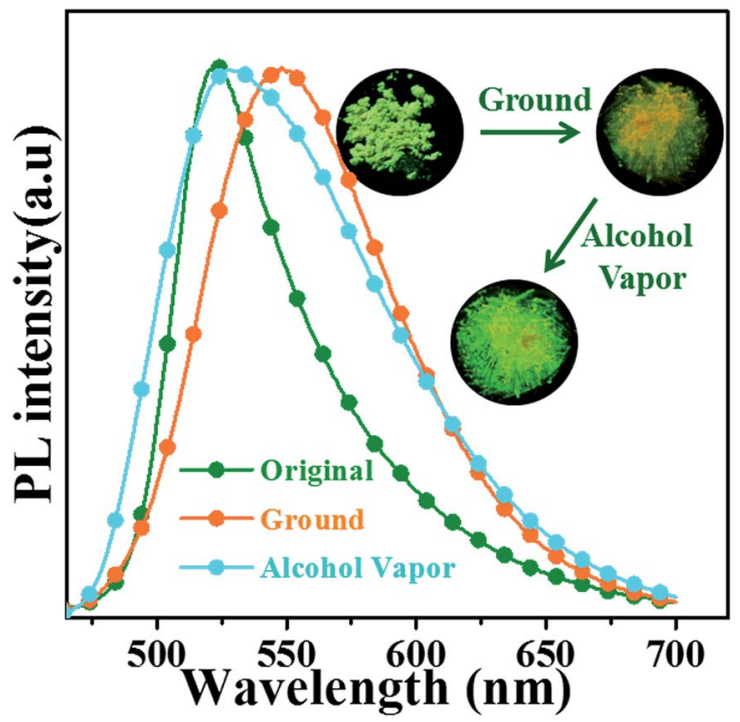

Fig. 8 (a) Photographs and PL spectra of oN-TPA in the crystalline powders, the ground powders, and then exposed to alcohol vapour.

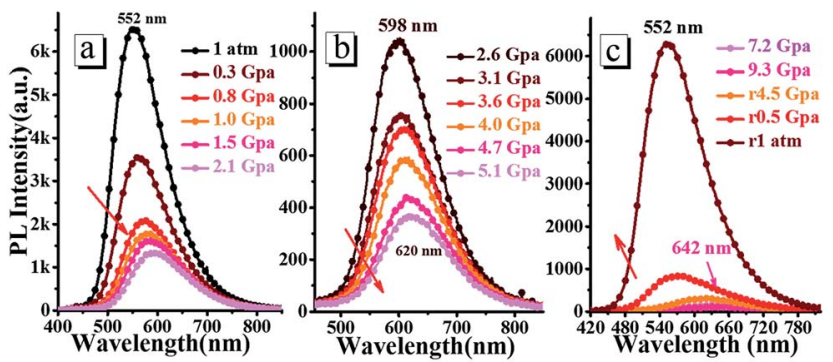

Fig. 9 Steady-state fluorescence spectra of the doped film under the different hydrostatic pressure during compression ( $a$ and $b$ ) and decompression (c).

3.5.2 High pressure experiments. The high pressure experiments of $\boldsymbol{o N}$-TPA were conducted by placing the doped film in the hole of a T301 steel gasket, using methanol and ethanol mixture $(4: 1)$ as a pressure-transmitting medium. As shown in Fig. 9, with the hydrostatic pressure gradually increased from 1 atm to $9.3 \mathrm{GPa}$, the fluorescence spectra demonstrate a remarkable red-shift from green $(552 \mathrm{~nm})$ to orange (598 nm), and further to red (642 nm). At the same time, the emission intensity is reduced continuously. In the nature light, the color of the doped film presents a change from green to red (Fig. S8 †). Upon the pressure released to $1 \mathrm{~atm}$, both color and fluorescence recover to the original state, indicating its good reversibility as a pressure sensor. The emissions can be switched between 552 and $642 \mathrm{~nm}$ (90 nm red-shift) during pressurizing-depressurizing process, which could be applied as piezochromic luminescent material.

\section{Conclusions}

In this work, we prepared a simple $\mathrm{D}-\pi-\mathrm{A}$ structure multistimuli responsive fluorescence molecule of $\boldsymbol{o N}$-TPA with AIEE effect. It exhibits obvious solvatochromic effect and its excited state is confirmed to be a HLCT state. Moreover, it can be used as a fast and effective PL probe to quantitatively detect low-level water in organic solvents, which is ascribed to the ICT effect with the increased mixture-solvents polarity. Because the pyridine unit can bind with a proton to form a cation, $\boldsymbol{o N}$-TPA can also be used as a sensor for detecting protonic acids both in solvent for TFA and in solid for $\mathrm{HCl}$ vapor. Finally, it exhibits pronounced mechanochromic fluorescence properties, the reversibly emissions can be switched between 552 and $642 \mathrm{~nm}$ (90 nm red-shift) during pressurizing-depressurizing process. This work not only comprehensively demonstrates the stimuliresponsive fluorescence behaviors of $\boldsymbol{o N}$-TPA, but also provides a $\mathrm{D}-\pi-\mathrm{A}$ structure luminescent material possessing potential applications in detection and sensing with remarkable PL changes.

\section{Conflicts of interest}

There are no conflicts to declare.

\section{Acknowledgements}

This work was supported by the National Natural Science Foundation of China (21171108) and Shanxi Provincial Fund for Natural Sciences (201601D011026).

\section{Notes and references}

1 (a) M. M. Caruso, D. A. Davis, Q. Shen, S. A. Odom, N. R. Sottos, S. R. White and J. S. Moore, Chem. Rev., 2009, 109, 5755-5798; (b) Z. Chi, X. Zhang, B. Xu, X. Zhou, C. Ma, Y. Zhang, S. Liu and J. Xu, Chem. Soc. Rev., 2012, 41, 3878-3896; (c) X. Zhang, Z. Chi, Y. Zhang, S. Liu and J. Xu, J. Mater. Chem. C, 2013, 1, 3376-3390; (d) H. Sun, S. Liu, W. Lin, K. Y. Zhang, W. Lv, X. Huang, F. Huo, H. Yang, G. Jenkins and Q. Zhao, Nat. Commun., 2014, 5, 3601.

2 (a) J. W. Chung, Y. You, H. S. Huh, B. K. An, S. J. Yoon, S. H. Kim, S. W. Lee and S. Y. Park, J. Am. Chem. Soc., 2009, 131, 8163-8172; (b) J. Wang, G. Yang, H. Jiang, G. Zou and Q. Zhang, Soft Matter, 2013, 9, 9785-9791.

3 (a) X. Luo, J. Li, C. Li, L. Heng, Y. Q. Dong, Z. Liu, Z. Bo and B. Z. Tang, Adv. Mater., 2011, 23, 3261-3265; (b) Y. Zhang, J. W. Sun, G. Zhuang, M. Ouyang, Z. W. Yu, F. Cao, G. X. Pan, P. S. Tang, C. Zhang and Y. G. Ma, J. Mater. Chem. C, 2014, 2, 195-200.

4 T. Guo, Q. Deng, G. Fang, Y. Yun, Y. Hu and S. Wang, Biosens. Bioelectron., 2016, 85, 596-602.

5 M. Zhang, S. Zheng, L. Ma, M. Zhao, L. Deng, L. Yang and L. J. Ma, Spectrochim. Acta, Part A, 2014, 124, 682-686.

6 (a) L. Sheng, M. Li, S. Zhu, H. Li, G. Xi, Y. Li, Y. Wang, Q. Li, S. Liang, K. Zhong and S. X.-A. Zhang., Nat. Commun., 2014, 5, 3044; (b) G. Xi, L. Sheng, I. Zhang, J. Du, T. Zhang, Q. Chen, G. Li, Y. Zhang, Y. Song, J. Li, Y. Zhang and S. X.-A. Zhang, ACS Appl. Mater. Interfaces, 2017, 9, 38032-38041.

7 T. Zhou, T. Jia, S. Zhao, J. Guo, H. Zhang and Y. Wang, Cryst. Growth Des., 2011, 12, 179-184. 
8 J. Luo, Z. Xie, J. W. Lam, L. Cheng, H. Chen, C. Qiu, H. S. Kwok, X. Zhan, Y. Liu and D. Zhu, Chem. Commun., 2001, 1740-1741.

9 (a) L. Yao, S. Zhang, R. Wang, W. Li, F. Shen, B. Yang and Y. Ma, Angew. Chem., 2014, 126, 2151-2155; (b) J. Huang, N. Sun, Y. Dong, R. Tang, P. Lu, P. Cai, Q. Li, D. Ma, J. Qin and Z. Li, Adv. Funct. Mater., 2013, 23, 2329-2337.

10 (a) S. Chen, J. Liu, Y. Liu, H. Su, Y. Hong, C. K. Jim, R. T. Kwok, N. Zhao, W. Qin, J. W. Lam, K. S. Wong and B. Z. Tang, Chem. Sci., 2012, 3, 1804-1809; (b) Y. Liu, Y. Tang, N. N. Barashkov, I. S. Irgibaeva, J. W. Lam, R. Hu, D. Birimzhanova, Y. Yu and B. Z. Tang, J. Am. Chem. Soc., 2010, 132, 13951-13953; (c) D. Ding, J. Liang, H. Shi, R. T. Kwok, M. Gao, G. Feng, Y. Yuan, B. Z. Tang and B. Liu, J. Mater. Chem. B, 2014, 2, 231-238; (d) Y. Zhang, S. Li, G. Pan, H. Yang, M. Qile, J. Chen, Q. Song and D. Yan, Sens. Actuators, B, 2018, 254, 785-794.

11 (a) M. J. Marín, F. Galindo, P. Thomas, T. Wileman and D. A. Russell, Anal. Bioanal. Chem., 2013, 405, 6197; (b) C. W. T. Leung, Y. Hong, S. Chen, E. Zhao, J. W. Y. Lam and B. Z. Tang, J. Am. Chem. Soc., 2013, 135, 62-65; (c) F. Oukacine, J. P. Quirino, L. Garrelly, B. Romestand, T. Zou and H. Cottet, Anal. Chem., 2011, 83, 4949-4954.

12 (a) J. Shi, S. Zhang, M. Zheng, Q. Deng, C. Zheng, J. Li and F. Huang, Sens. Actuators, B, 2017, 238, 765-771; (b) S. Umar, A. K. Jha, D. Purohit and A. Goel, J. Org. Chem., 2017, 82, 4766-4773; (c) Y. Zhang, Q. Song, K. Wang, W. Mao, F. Cao, J. Sun, L. Zhan, Y. Lv, Y. Ma, B. Zou and C. Zhang, J. Mater. Chem. C, 2015, 3, 3049-3054.

13 (a) C. Feng, K. Wang, Y. Xu, L. Liu, B. Zou and P. Lu, Chem. Commun., 2016, 52, 3836-3839; (b) G. Yin, Y. Ma, Y. Xiong, X. Cao, Y. Li and L. Chen, J. Mater. Chem. C, 2016, 4, 751-757.

14 (a) W. Li, Y. Pan, L. Yao, H. Liu, S. Zhang, C. Wang, F. Shen, P. Lu, B. Yang and Y. Ma, Adv. Opt. Mater., 2014, 2, 892-901; (b) H. Uoyama, K. Goushi, K. Shizu, H. Nomura and C. dachi, Nature, 2012, 492, 234.
15 (a) C. Ramanan, A. L. Smeigh, J. E. Anthony, T. J. Marks and M. R. Wasielewski, J. Am. Chem. Soc., 2011, 134, 386-397; (b) X. Zhang, Z. Lu, L. Ye, C. Zhan, J. Hou, S. Zhang, B. Jiang, Y. Zhao, J. Huang and S. Zhang, Adv. Mater., 2013, 25, 5791-5797.

16 (a) A. Pron, P. Gawrys, M. Zagorska, D. Djurado and R. Demadrille, Chem. Soc. Rev., 2010, 39, 2577-2632; (b) X. Zhan, A. Facchetti, S. Barlow, T. J. Marks, M. A. Ratner, M. R. Wasielewski and S. R. Marder, Adv. Mater., 2011, 23, 268-284.

17 (a) Y. Jiang, Y. Wang, J. Hua, J. Tang, B. Li, S. Qian and H. Tian, Chem. Commun., 2010, 46, 4689-4691; (b) B. Wang, Y. Wang, J. Hua, Y. Jiang, J. Huang, S. Qian and H. Tian, Chem.-Eur. J., 2011, 17, 2647-2655.

18 (a) W. Li, Y. Pan, R. Xiao, Q. Peng, S. Zhang, D. Ma, F. Li, F. Shen, Y. Wang, B. Yang and Y. Ma, Adv. Funct. Mater., 2014, 24, 1609-1614; (b) H. Liu, Q. Bai, L. Yao, H. Zhang, H. Xu, S. Zhang, W. Li, Y. Gao, J. Li, P. Lu, H. Wang, B. Yang and Y. Ma, Chem. Sci., 2015, 6, 3797-3804; (c) S. Zhang, L. Yao, Q. Peng, W. Li, Y. Pan, R. Xiao, Y. Gao, C. Gu, Z. Wang, P. Lu, F. Li, S. Su, B. Yang and Y. Ma, Adv. Funct. Mater., 2015, 25, 1755-1762; (d) Y. Zhang, K. Wang, G. Zhuang, Z. Xie, C. Zhang, F. Cao, G. Pan, H. Chen, B. Zou and Y. Ma, Chem.-Eur. J., 2015, 21, 2474.

19 Z. R. Grabowski, K. Rotkiewicz and W. Rettig, Chem. Rev., 2003, 103, 3899-4031.

20 Y. Zhang, M. Qile, J. Sun, M. Xu, K. Wang, F. Cao, W. Li, Q. Song, B. Zou and C. Zhang, J. Mater. Chem. C, 2016, 4, 9954.

21 S. Zhang, W. Li, L. Yao, Y. Pan, F. Shen, R. Xiao, B. Yang and Y. Ma, Chem. Commun., 2013, 49, 11302-11304.

22 R. L. Martin, J. Chem. Phys., 2003, 118, 4775-4777.

23 (a) P. S. Hariharan, J. Pitchaimani, V. Madhu and S. P. Anthony, Opt. Mater., 2017, 64, 53-57; (b) P. S. Hariharan, E. M. Mothi, D. Moon and S. P. Anthony, ACS Appl. Mater. Interfaces, 2016, 8, 33034-33042. 